\title{
Meta-Analysis of Chinese Traditional Medicine Bushen Huoxue Prescription for Endometriosis Treatment
}

\author{
Jing Shan, ${ }^{1,2}$ Wen Cheng, ${ }^{2}$ Dong-xia Zhai, ${ }^{2}$ Dan-ying Zhang, ${ }^{2}$ Rui-pin Yao, ${ }^{2}$ Ling-ling Bai, \\ Zai-long Cai, ${ }^{3}$ Yu-huan Liu, ${ }^{4}$ and Chao-qin $\mathbf{Y u}^{2}$ \\ ${ }^{1}$ Shanghai University of Traditional Chinese Medicine, Shanghai, China \\ ${ }^{2}$ Department of Gynecology of Traditional Chinese Medicine, Changhai Hospital, Second Military Medical University, Shanghai, China \\ ${ }^{3}$ Department of Biochemistry and Molecular Biology, Second Military Medical University, Shanghai, China \\ ${ }^{4}$ Department of Gynecology and Obstetrics, Changhai Hospital, Second Military Medical University, Shanghai, China
}

Correspondence should be addressed to Yu-huan Liu; 13651968369@163.com and Chao-qin Yu; chqyu81@163.com

Received 9 July 2017; Accepted 20 September 2017; Published 23 November 2017

Academic Editor: Hyunsu Bae

Copyright (C) 2017 Jing Shan et al. This is an open access article distributed under the Creative Commons Attribution License, which permits unrestricted use, distribution, and reproduction in any medium, provided the original work is properly cited.

\begin{abstract}
Objectives. To evaluate the efficacy and safety of Bushen Huoxue prescription (BSHXP) for endometriosis. Methods. A meta-analysis was performed, and studies were searched from the seven databases from the date of database establishment to April 30, 2017. Randomized controlled trials (RCTs) that explored the efficacy and safety of BSHXP for patients with endometriosis were included. Two assessors independently reviewed each trial. The Cochrane Risk of Bias assessment tool was used for quality assessment. Results. In the 13 included studies, the total effectiveness rates of BSHXP were higher than those of Western medicine (RR, 1.55; 95\% CI, 1.03-2.32; $P=0.04$ ), but the dysmenorrhea alleviation rates of the two treatments did not significantly differ (RR, 1.28; 95\% CI, 0.70-2.34; $P=0.42$ ). The pregnancy rates of BSHXP were also higher than those of hormone therapy $(\mathrm{RR}, 1.99 ; 95 \%$ CI, 1.17-3.39; $P=0.01$ ). However, whether BSHXP is more effective than Western medicine in diminishing endometriotic cyst remains unknown. Conclusions. Our study provides evidence that BSHXP is effective and safe for endometriosis, but this evidence is inconclusive because of the low methodological quality of the included RCTs. Our findings suggest that BSHXP is an alternative drug for endometriosis, but it should be further examined in future clinical research.
\end{abstract}

\section{Introduction}

Endometriosis is a gynecological disease characterized by the presence and growth of ectopic endometrial tissue and often associated with inflammation, chronic pain, and infertility [1]. Compared with normal females, affected women are at a twofold to threefold increased risk of developing ovarian cancer, although endometriosis is a seemingly benign disease [2]. The prevalence of endometriosis approximately ranges from $2 \%$ to $10 \%$ of reproductive aged women and to $50 \%$ of infertile women [3,4]. Endometriosis can significantly influence the quality of life, ability to work, and fertility of reproductive aged women [5]. However, endometriosis is poorly understood, and the incidence of endometriosis likely increases [6]. The definitive treatment for endometriosis remains a difficult problem [7]. Current treatment strategies aim to diminish lesions, reduce pain, improve reproductive capacity, and reduce and avoid the chance of recurrence [8]. Various treatment methods, including medical, surgical, and alternative approaches, have been administered to patients with endometriosis [9]. Medications used in clinical settings commonly include hormonal contraceptives, GnRH agonists, aromatase inhibitors, nonsteroidal anti-inflammatory drugs, and progestins [10]. These medications can alleviate the symptoms of endometriosis, but these benefits disappear upon drug discontinuation [11]. Most of these options yield some side effects, such as insomnia, weight gain, rash, mood swings, depression, nausea, headache, bone density loss, and venous thrombosis [12]. Considering that endometriosis is a chronic condition, researchers should develop medical therapy that can be used continuously for symptom control [13]. Alternative therapies for endometriosis have also 
TABLE 1: Evaluation criteria on the efficacy of TCM symptom and syndromes recommended by GCRNDTCM.

\begin{tabular}{ll}
\hline Classification & Detailed description \\
\hline \multirow{2}{*}{ Cure } & (A) Clinical symptoms, gynecological examination, and B-ultrasound pelvic mass disappeared \\
Markedly & (B) No recurrence was found within 6 months \\
& Clinical symptoms disappeared or significantly reduced, with gynecological examination and \\
Effective & B-ultrasound pelvic mass reduction of $2 / 3$ or more \\
& Clinical symptoms, gynecological examination, and B-ultrasound examination pelvic mass \\
& reduction $1 / 3$ or more \\
Invalid & (A) Clinical symptoms, gynecological examination, and B-ultrasound pelvic mass had no \\
& significant change \\
& (B) Recurrence of progressive abdominal pain, and B-ultrasound reemergence of pelvic mass or \\
& mass has been reduced to grow up again
\end{tabular}

TCM, traditional Chinese medicine; GCRNDTCM, Guidelines of Clinical Research of New Drugs of Traditional Chinese Medicine.

been developed. Among various therapies for endometriosis, traditional Chinese medicine (TCM) has been preferred by women with endometriosis [14].

According to TCM theory, kidney deficiency and blood stasis are a common syndrome of endometriosis, which is usually characterized by various conditions, such as dysmenorrhea, back pain, blood clots in menstruation, cold hands and feet, and fatigue [15]. Endometriosis is effectively treated with Bushen Huoxue prescription (BSHXP). In vivo tests have shown that BSHXP can inhibit the invasion of endometrial stromal cells and the expression of MMP-9 [16]. Animal experiments have demonstrated that BSHXP can promote the ability of apoptotic cells in endometriosis to hasten the atrophy and regression of endometriotic tissues [17].

However, data supporting the validity of this treatment are insufficient. This systematic review aimed to evaluate the efficacy and safety of BSHXP as a treatment for endometriosis by integrating different outcomes from clinical studies.

\section{Methods and Materials}

2.1. Methods. This systematic review was performed according to the Preferred Reporting Items for Systematic Review and Meta-Analyses Statement [18].

\subsection{Study Selection}

2.2.1. Types of Studies. All of the RCTs reporting the application of BSHXP for the treatment of endometriosis were included. No limitations on publication status or language were set. Non-RCTs or animal studies were excluded.

2.2.2. Types of Participants. Only female patients who satisfied at least one of the current diagnostic standards of endometriosis and kidney deficiency and blood stasis syndrome were included. No limitations on ethnicity or nationality were set. Patients with adenomyosis were excluded.

2.2.3. Types of Interventions. RCTs that observed and compared the effects of BSHXP and Western medicine were identified. Patients in the treatment group were given a kidney-tonifying and blood-activating formula. Patients in the control group were treated with Western medicine. Patients were excluded when the trials included other cointerventions, including another herbal formula. We did not set limitations on dosages and routes of the administration of the traditional Chinese herbs.

2.2.4. Types of Outcome Measures. The primary outcomes in this study were the total effectiveness rate, dysmenorrhea alleviation rate, and pregnancy rate. The secondary outcomes were changes in the size of endometriotic cyst and adverse events. The total effectiveness rate was assessed according to the evaluation criteria of the Guidelines of Clinical Research of New Drugs of Traditional Chinese Medicine (Table 1).

2.3. Search Strategy. Electronic searches were conducted in the Cochrane Central Register of Controlled Trials (CENTRAL, 1996-April 2017), EMBASE (1966-April 2017), PubMed (1959-April 2017), the Chinese National Knowledge Infrastructure (CNKI, 1979-April 2017), the Wanfang Database (1959-April 2017), the Chinese Scientific Journal Database (VIP, 1989-April 2017), and the Chinese Biomedical Literature Database (CBM, 1978-April 2017) from the date of database establishment to April 30, 2017, by two reviewers (J. Shan and W. Cheng). The references of the identified studies and ongoing registered clinical trials were also manually searched to retrieve unpublished articles. No restriction on language or publication status was set. The search terms for the literature search were as follows: ("endometriosis" OR "dysmenorrhea" OR "dyspareunia" OR "pelvic pain”) AND ("tonifying kidney and activating blood" OR "bu shen" OR "wen shen" OR "zi shen" OR "huo xue" OR "qu yu” OR "xiao zheng”) AND ("clinical trial” OR "randomized controlled trial” OR "randomised controlled trial”).

2.4. Data Extraction. Two reviewers (Dong-xia Zhai and Dan-ying Zhang) extracted basic information independently by using a standardized data extraction form. Disagreements were resolved by discussion or consensus with a third reviewer (Chao-qin Yu). Some important data, including first author's name, publication year, sample size, treatment interventions and control groups, BSHXP composition, treatment duration, outcome measures, and adverse effects, were extracted from primary trials. If a study was incomprehensive 
or uncertain, we then contacted the corresponding author by telephone, email, and fax to obtain the correct data.

2.5. Assessment of Risk of Bias. The risk of bias of the eligible trials was assessed by two independent authors using the Cochrane Risk of Bias tool [19]. Disagreements were resolved by obtaining consensus with a third reviewer (Chao-qin $\mathrm{Yu}$ ). The risk of bias was evaluated on the basis of these domains: (1) selection bias (adequate sequence generation and concealment of allocation); (2) performance bias (blinding of the investigator and blinding of the assessor); (3) detection bias (blinding of the assessor); (4) attrition bias (incomplete outcome data addressed); (5) reporting bias (free of selective reporting); and (6) other sources of bias (other potential threats to validity).

2.6. Data Analyses. BSHXP and Western medicine were compared in this review. Outcome measures after treatment were presented as risk ratio (RR) with $95 \%$ CI for dichotomous outcomes. Cochrane's $P$ values and $I^{2}$ tests were determined to examine the level of heterogeneity between trials. A random-effects model was used to evaluate the effects of BSHXP on endometriosis if $I^{2}>50 \%$ or $P<0.1$. Otherwise, a fixed-effects model was utilized. $P<0.05$ was considered statistically significant. Data were subjected to meta-analysis by using Review Manager 5.3 (Cochrane Community, London, United Kingdom, 2014).

\section{Results}

3.1. Study Selection. A total of 146 studies were collected through document retrieval. Of these studies, 12 duplicate publications were removed. After their titles and abstracts were scanned, 77 articles were also excluded because they were clinical experience reports, case studies, and animal research. The 28 remaining full-text articles were further analyzed, but 15 trials were also eliminated because of the following: 2 non-RCT studies, 3 articles with duplicate publication of data, 4 articles with mixed interventions, and 6 articles with a control group containing herbal therapies. Finally, 13 eligible studies involving 936 patients with endometriosis were included in our meta-analysis (Figure 1).

3.2. Study Characteristics. The basic information of the included RCTs is summarized in Table 2. This meta-analysis included 13 trials. Of these trials, 12 were published in Chinese and 1 was presented in English. Of the 936 patients with endometriosis enrolled in these trials, 492 were assigned to the treatment group and 444 were designated in the control group. The sample sizes of these trials ranged from 48 to 120 . The baseline in all of the trials did not significantly differ.

All of the trials involved two-arm designs: treatment group versus control group. Patients in the treatment group were treated with BSHXP (Table 3), while patients in the control group were administered with Western medicine, including mifepristone, Diphereline, gestrinone, and danazol. Treatment duration was 3 or 6 months. Of the 13 trials, 3 indicated dropouts $[23,29,30] ; 10$ reported the total effective rates [21-30]; 7 presented the dysmenorrhea alleviation rates

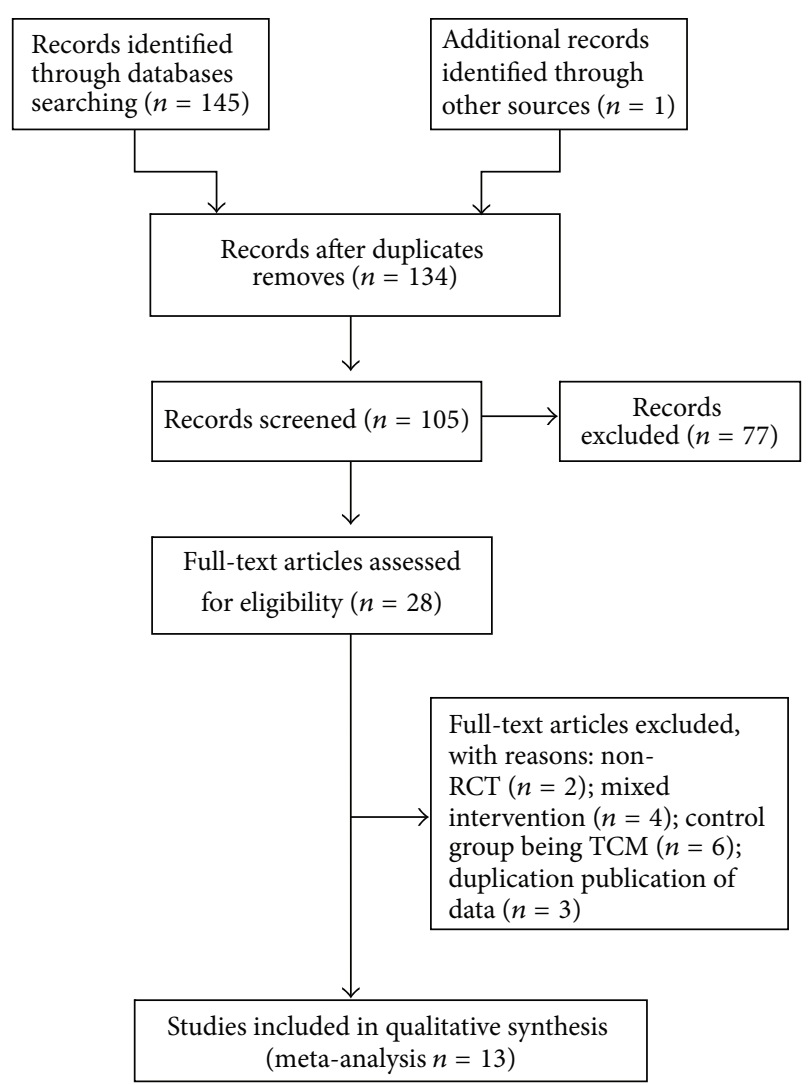

FIGURE 1: Flow diagram of study selection and identification.

$[24,25,27,29-32] ; 3$ described the pregnancy rates [20,31, $32]$; and 6 discussed adverse effects [20, 21, 24, 29, 30, 32].

3.3. Risk of Bias Assessment. In Figure 2, the methodology quality of the included trials was assessed as low. Although all of the trials were randomized, 3 reported the method of generating a random sequence (random number table) [2123]. None of the trials reported any concealed allocation or blinding of patients and study participants. Of the 13 trials, 2 provided the number and reasons of dropouts [22,24]. All of the relevant trials adequately addressed incomplete outcome data and selective reporting, and 4 trials included follow-up that ranged from 6 months to 1 year $[20-22,24]$.

\section{Outcome Measures}

4.1. Total Effectiveness Rates. In 10 studies, 633 patients with endometriosis were included. The experimental and control groups received BSHXP and Western medicine, respectively. Heterogeneity tests involved a fixed-effects model to describe the data of the total effectiveness $\left(\chi^{2}=8.18 ; P=0.52 ; I^{2}=\right.$ $0 \%)$. Our meta-analysis confirmed that the total effective rate of the BSHXP group was higher than that of the control group (RR, 1.55; 95\% CI, 1.03-2.32; $P=0.04$, Figure 3(a)).

4.2. Dysmenorrhea Alleviation Rates. In 7 studies, the effects of BSHXP and Western medicine on dysmenorrhea were compared and evaluated. Of the 314 patients with 


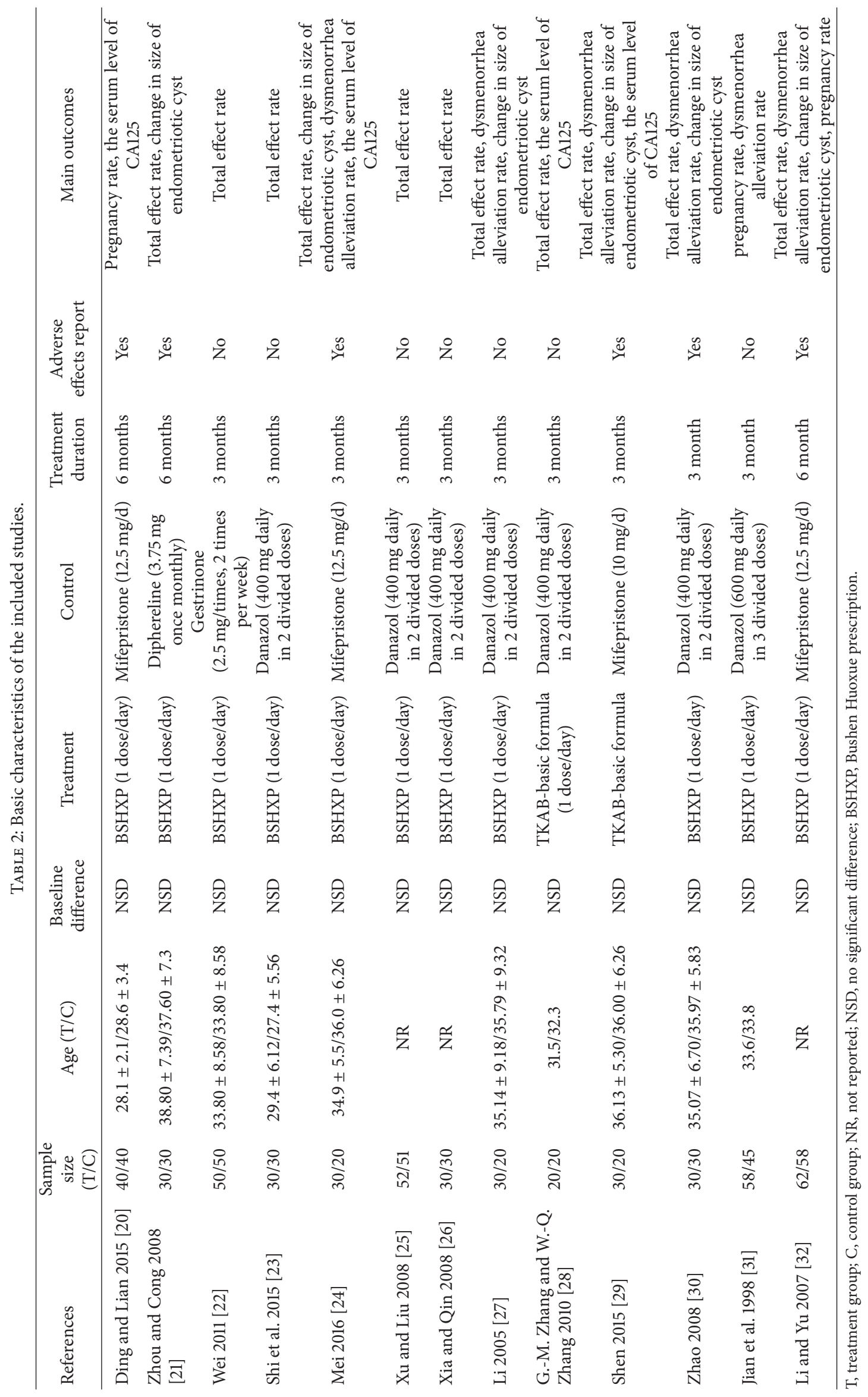


TABLE 3: Herbal medicines in the included studies.

\begin{tabular}{|c|c|c|}
\hline References & Formula & Composition of formula \\
\hline Ding and Lian 2015 [20] & BSHXP & $\begin{array}{l}\text { Prepared Radix Rehmanniae } 20 \text { g, Dodder } 20 \text { g, Angelica } 15 \text { g, Salvia } 15 \text { g, } \\
\text { Caulis Spatholobi } 15 \text { g, Cyperus rotundus } 20 \text { g, Curcuma zedoaria } 12 \text { g, } \\
\text { Chuan Cattle Cane } 12 \text { g, Poria Cocos } 15 \text { g, Cassia Twig } 15 \text { g, Rhizoma } \\
\text { Corydalis } 15 \text { g, Trogopterus Dung } 15 \text { g, Red Peony Root } 15 \text { g }\end{array}$ \\
\hline Zhou and Cong 2008 [21] & BSHXP & $\begin{array}{l}\text { Prunella vulgaris } 30 \mathrm{~g} \text {, Raw Oyster } 30 \mathrm{~g} \text {, Calcined Corrugated Child } 30 \mathrm{~g} \text {, } \\
\text { Salvia chinensis } 18 \mathrm{~g} \text {, Codonopsis } 15 \mathrm{~g} \text {, Sunburn Turtle } 12 \mathrm{~g} \text {, Antler Slices } \\
9 \mathrm{~g} \text {, Eupolyphaga } 9 \mathrm{~g} \text {, Leeches } 6 \mathrm{~g} \text {, Gun Ginger } 6 \mathrm{~g}\end{array}$ \\
\hline Wei 2011 [22] & BSHXP & $\begin{array}{l}\text { Astragalus } 20 \mathrm{~g} \text {, White Atractylodes Rhizome } 10 \mathrm{~g} \text {, Chinese Yam } 15 \mathrm{~g} \text {, } \\
\text { Dipsacus } 10 \mathrm{~g} \text {, Dodder } 10 \mathrm{~g} \text {, Psoralen } 10 \mathrm{~g} \text {, Angelica } 15 \mathrm{~g} \text {, Red Peony } 10 \mathrm{~g} \text {, } \\
\text { Safflower } 10 \mathrm{~g} \text {, Triangular } 10 \mathrm{~g} \text {, Curcuma } 10 \mathrm{~g} \text {, Pollen Typhae } 10 \mathrm{~g}, \\
\text { Trogopterus Dung } 10 \mathrm{~g}\end{array}$ \\
\hline Shi et al. 2015 [23] & BSHXP & $\begin{array}{l}\text { Triad } 9 \mathrm{~g} \text {, Curcuma } 9 \mathrm{~g} \text {, Pilgrimage } 12 \mathrm{~g} \text {, Eupolyphaga } 12 \mathrm{~g} \text {, Cistanche } \\
12 \mathrm{~g} \text {, Cuscuta } 12 \mathrm{~g} \text {, Morinda } 12 \mathrm{~g} \text {, Cynomorium } 12 \mathrm{~g} \text {, Tortoise Plastron } \\
18 \mathrm{~g} \text {, Antler Slices } 9 \mathrm{~g} \text {, Aerial Parts of Epimedium } 30 \mathrm{~g} \text {, Hematoxylin } 9 \mathrm{~g} \text {, } \\
\text { Prunella vulgaris } 9 \mathrm{~g}\end{array}$ \\
\hline Mei 2016 [24] & BSHXP & $\begin{array}{l}\text { Dodder } 10 \mathrm{~g} \text {, Placenta } 10 \mathrm{~g} \text {, Angelica } 10 \mathrm{~g} \text {, Szechuan Lovage Root } 10 \mathrm{~g} \text {, } \\
\text { Citri Reticulatae Viride } 6 \mathrm{~g} \text {, Red Peony } 10 \mathrm{~g} \text {, Corydalis Rhizome } 10 \mathrm{~g} \text {, } \\
\text { Euonymus alatus } 10 \mathrm{~g}\end{array}$ \\
\hline $\mathrm{Xu}$ and Liu 2008 [25] & BSHXP & $\begin{array}{l}\text { Morinda } 20 \mathrm{~g} \text {, Aerial Parts of Epimedium } 20 \mathrm{~g} \text {, Chinese yam } 15 \mathrm{~g} \text {, Cornus } \\
\text { officinalis } 15 \mathrm{~g} \text {, Rehmannia } 15 \mathrm{~g} \text {, Motherwort } 15 \mathrm{~g} \text {, Corydalis Rhizome } \\
15 \mathrm{~g} \text {, Combined Spicebush Root } 15 \mathrm{~g} \text {, Rhizoma Sparganii } 10 \mathrm{~g} \text {, Curcuma } \\
\text { zedoaria } 10 \mathrm{~g}\end{array}$ \\
\hline Xia and Qin 2008 [26] & BSHXP & $\begin{array}{l}\text { Dodder } 20 \text { g, Eucommia } 15 \text { g, Astragalus } 40 \text { g, Salvia } 20 \text { g, Cinnamon } \\
6 \text { g, Red Peony } 12 \text { g, Trogopterus Dung15 g, Peach Kernel } 9 \text { g, Cyperus } \\
\text { rotundus } 12 \text { g, Chicken Gizzard's Internal Lining } 6 \text { g, Poria } 12 \text { g, Cedar } \\
12 \text { g, licorice } 6 \text { g. }\end{array}$ \\
\hline Li 2005 [27] & BSHXP & $\begin{array}{l}\text { Dodder } 30 \text { g, Astragalus } 30 \text { g, Salvia } 30 \text { g, Eucommia } 15 \text { g, Cinnamon } 6 \text { g, } \\
\text { Red Peony } 12 \text { g, Trogopterus Dung } 15 \text { g, Peach Kernel } 9 \text { g, Tree Peony } \\
\text { Bark } 12 \text { g, Nut Grass Rhizome } 12 \text { g, Chicken Gizzard's Internal Lining } \\
6 \text { g, Poria } 12 \text { g, Liquorice Root } 6 \text { g }\end{array}$ \\
\hline $\begin{array}{l}\text { G.-M. Zhang and W.-Q. } \\
\text { Zhang } 2010 \text { [28] }\end{array}$ & BSHXP & $\begin{array}{l}\text { Angelica } 10 \mathrm{~g} \text {, Red Peony } 19 \mathrm{~g} \text {, Poria } 10 \mathrm{~g} \text {, Atractylodes } 10 \mathrm{~g} \text {, Chinese Yam } \\
10 \mathrm{~g} \text {, Trogopterus Dung } 10 \mathrm{~g} \text {, Eucommia } 12 \mathrm{~g} \text {, Dodder } 12 \mathrm{~g} \text {, Antler Cream } \\
12 \mathrm{~g} \text {, Corydalis Rhizome } 15 \mathrm{~g} \text {, Pangolin } 1.5 \mathrm{~g}\end{array}$ \\
\hline
\end{tabular}

endometriosis included in these studies, 167 were included in the BSHXP group and 147 were assigned in the Western medicine group. The pooled results from these trials did not significantly differ in terms of the dysmenorrhea alleviation rates between the two groups (RR, 1.28; 95\% CI, 0.70-2.34; $P=0.42$, Figure 3(b)).

4.3. Pregnancy Rates. In 3 trials, pregnancy rates were reported. A total of 114 patients were included in the BSHXP group and 103 patients were assigned to the control group. Our meta-analysis showed that the pregnancy rates of the BSHXP group were higher than those of the Western medicine group (RR, 1.99; 95\% CI, 1.17-3.39; $P=0.01$, Figure 3(c)).

4.4. Change in Size of Endometriotic Cysts. In 6 studies, changes in the size of ectopic cysts were described. Three studies [24, 27, 29] compared the maximum cross-sectional areas of the cysts; two studies [20, 30] compared the maximum diameter line; one study [32] compared the reduction rates. Data could not be analyzed statistically because of inconsistent measurement standards. Thus, we only described the results of the original study.

In 2 studies [24, 29], the sizes of the cyst before and after treatment between BSHXP- and Western medicine-treated groups did not significantly differ $(P>0.05)$. Also the sizes of the cysts between the two groups did not also significantly vary after treatment $(P>0.05)$. In 4 studies [20, 27, 30, 32], the size of the cysts significantly differed $(P<0.05)$ before and after treatment between BSHXP- and Western medicinetreated groups. Nevertheless, the sizes of the cysts in these two groups did not significantly differ after treatment $(P>0.05)$.

Thus, our findings failed to support that TCM was more effective than Western medicine in reducing the size of endometriotic cysts.

4.5. Adverse Events. Six trials reported adverse events (6/14, $42.86 \%$ ) [20, 21, 24, 29, 30, 32], whereas the other seven trials mentioned no significant adverse reactions (8/14, 57.14\%) [21, $23,25-28,31]$. In six trials, no evident adverse events occurred in the BSHXP group. In three trials, seven patients in the Western medicine group suffered from liver dysfunction with 


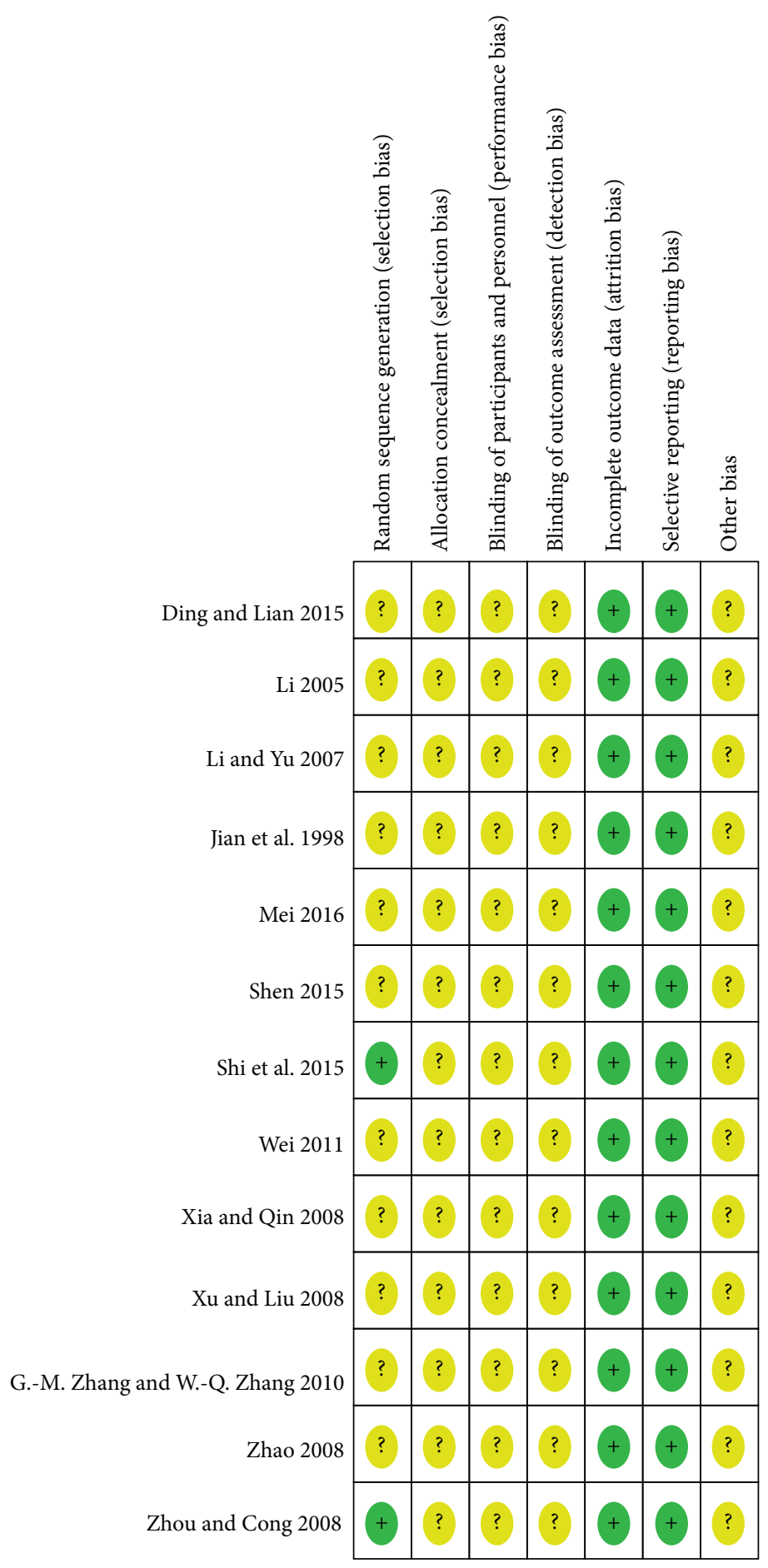

FIGURE 2: Risk of bias summary. +: low risk; -: high risk; ?: unclear risk.

increased alanine transaminase (ALT) levels (10/102, 9.80\%) $[24,29,30]$. In two trials $[20,30], 14$ patients experienced hot flashes $(14 / 60,23.33 \%)$. In one trial [30], six patients had amenorrhea $(6 / 30,20.00 \%)$. In one trial [30], seven patients manifested acne $(11 / 62,17.74 \%)$. In one trial [21], two patients exhibited mood changes $(2 / 30,6.67 \%)$; three patients suffered from vaginal dryness $(3 / 30,10.00 \%)$, and three patients had decreased libido $(3 / 30,10.00 \%)$.

4.6. Publication Bias. The funnel plot of the 10 trials that compared BSHXP with Western medicine in terms of their total effective rates was analyzed to detect possible 


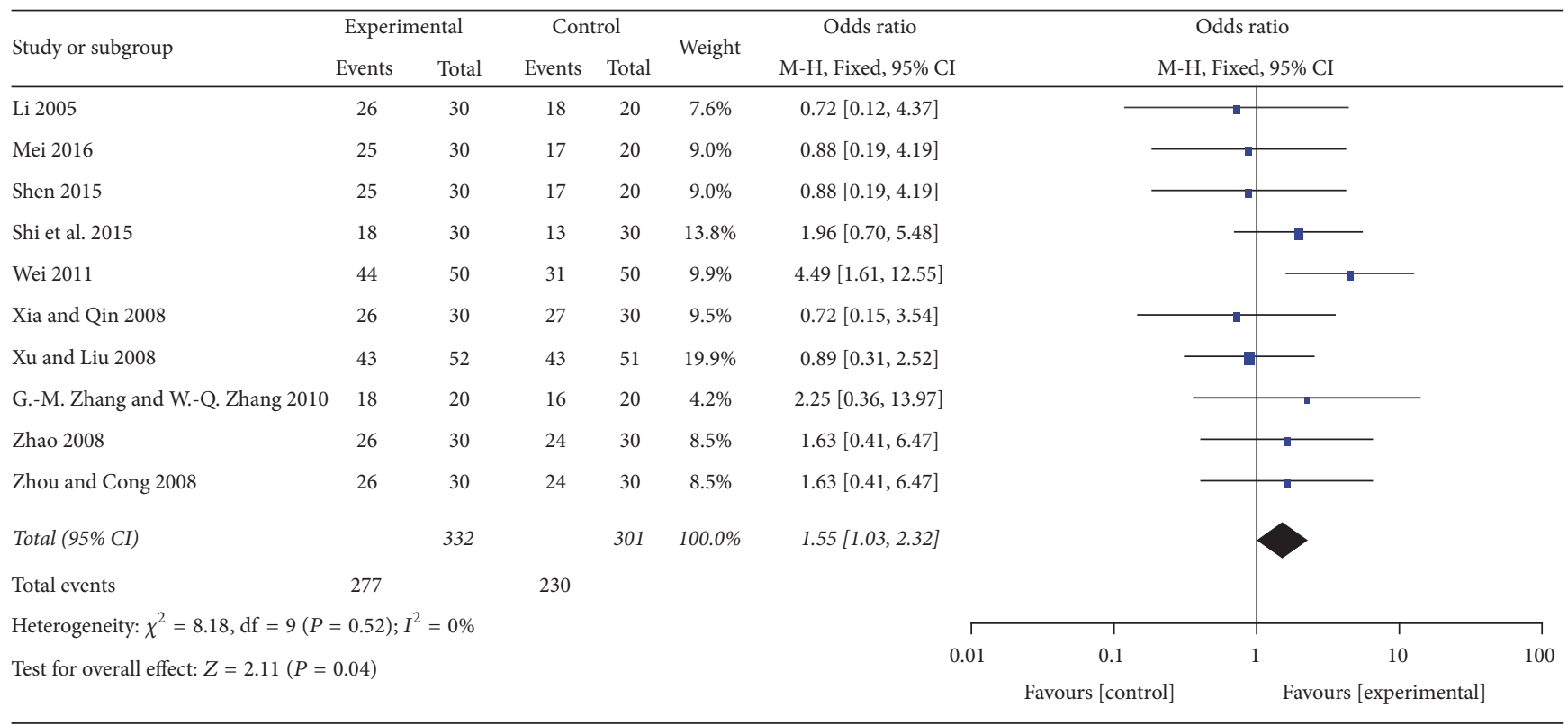

(a) Total effectiveness rates

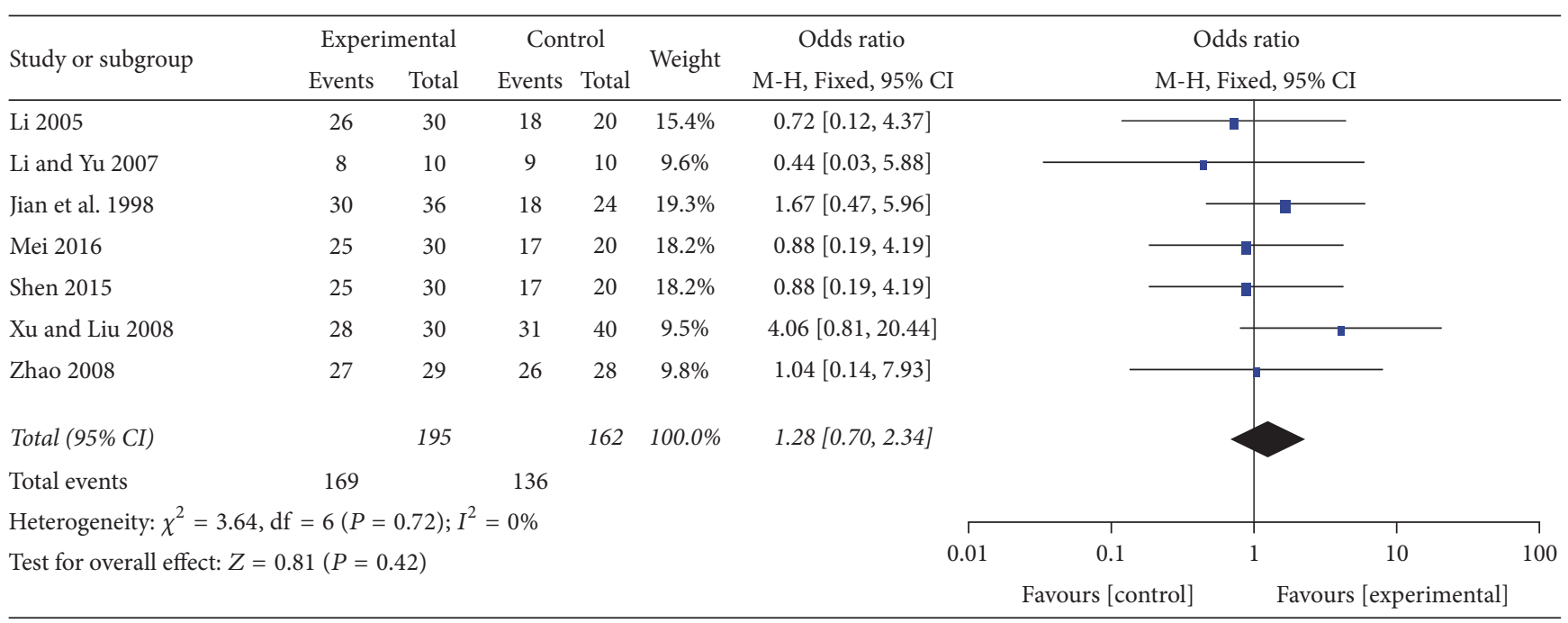

(b) Dysmenorrhea alleviation rates

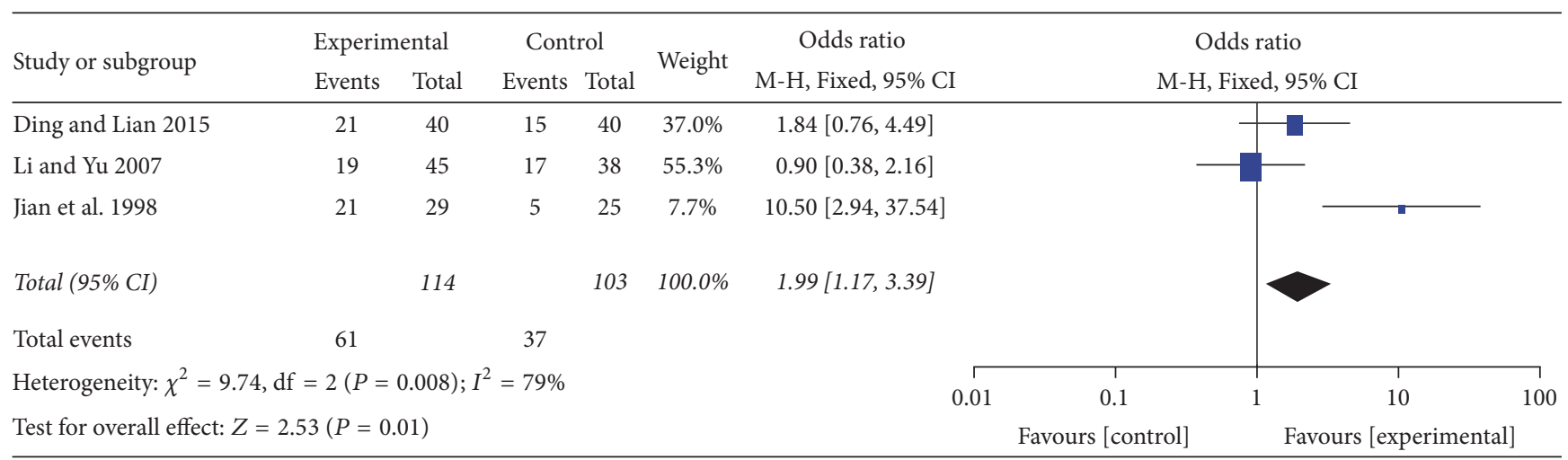

(c) Pregnancy rates

FIGURE 3: Meta-analysis of the total effectiveness rate, dysmenorrhea alleviation rate, and pregnancy rate of Bushen Huoxue prescription (BSHXP) versus Western medicine. 


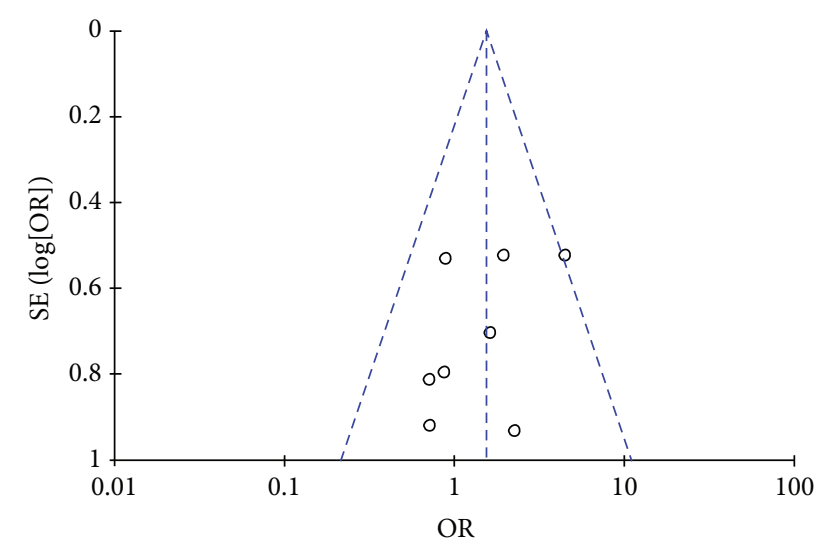

FIGURE 4: Funnel plot of the comparison of BSHXP versus Western medicine for the outcome of the total effectiveness rate.

publication bias. Figure 4 shows an asymmetrical funnel plot that indicates publication bias in the 10 selected articles.

\section{Discussion}

5.1. Summary of Evidence. A total of 936 patients with endometriosis were collected from the 13 RCTs in the review. The baseline of each study was consistent, and no statistical heterogeneity was observed. Our meta-analysis obtained the following results: (1) BSHXP did not significantly differ from Western medicine in terms of improving endometriosisrelated symptoms and syndromes. (2) Similar to Western medicine, BSHXP emerged as an effective alternative therapy to alleviate endometriosis-associated pain. (3) BSHXP could significantly enhance the clinical pregnancy rate, but this conclusion should be further verified in future studies. (4) Studies have yet to provide evidence supporting that BSHXP is more effective than Western medicine in terms of reducing the size of endometriotic cysts. (5) The adverse effects of BSHXP were less reported than those of Western medicine, although six trials described serious events. Considering the number of the analyzed RCTs and the high risk of bias, we experienced difficulty in obtaining our conclusions.

5.2. Promising Alternative Therapy for Endometriosis. Longterm therapy is required for patients diagnosed with endometriosis. Current medical treatments may elicit side effects, such as inhibiting ovulation and menstruation and reducing estradiol to postmenopausal levels, because a definitive treatment for endometriosis has yet to be established [33, 34]. However, the natural history of this disease remains poorly understood [34]. As a chronic condition, endometriosis threatens the health of patients and entails high cost of care $[35,36]$. Medical treatment should completely cure endometriosis rather than provide temporary relief from its symptoms [37]. In terms of endometriosis-associated infertility, new therapies have been developed to control pain symptoms without inhibiting ovulation [38]. New drugs replacing hormonal medicine for endometriosis will be available in the future, and these therapies should provide a normal and safe option for infertility [39]. In this regard, Chinese herbal medicines as complementary medicines are of great interest. Traditional Chinese medicine has been used to treat endometriosis. Tonifying kidney and activating blood stasis therapy may be a promising approach to treat endometriosis.

Considering syndrome differentiation and treatment system, doctors believe that endometriosis is mostly caused by kidney deficiency and blood stasis. Tonifying kidney and activating blood stasis prescriptions are designed. According to TCM theory, kidneys store essence and dominate reproduction. Chinese nourishing kidney herbs can regulate the hypothalamus-pituitary-ovary axis in dual directions and improve the functions of ovaries [40]. Liuwei Dihuang decoction, a well-known kidney-tonifying recipe, can enhance the rates of high-quality oocytes and embryos by regulating the microenvironment for oocytes of patients with endometriosis [41]. Ectopic cysts induce periodical bleeding defined in Chinese medicine as abnormal blood flow causing blood stasis. Activating blood herbs can promote blood circulation and alleviate blood stasis. Its treatment mechanism may be related to the regulation of immune conditions and the promotion of cell apoptosis in ectopic endometrium in vivo [42-44]. However, valid evidence, including meta-analysis results, has yet to be obtained for further recommendation. To our knowledge, this meta-analysis is the first research written in English to examine the effectiveness of BSHXP for endometriosis treatment.

\section{Limitations}

The methodologic quality of this review was generally poor. Although all of the studies were randomized, only two trials involved sequence generation [21,23]. No trials reported allocation concealment. If patients and implementers were aware of the interventions, this meta-analysis would directly result in performance and detection biases. Seven trials reported adverse events, and these adverse events were described briefly. Therefore, the conclusion about the safety of BSHXP could not be obtained definitely.

This meta-analysis was also limited by various evaluating criteria among different studies. For example, three trials compared the maximum cross-sectional areas in terms of the size of cysts, two trials compared the maximum diameter, and one trial indicated the reduction ratio. Thus, we were unable to analyze these data uniformly. We suggest that researchers should use internationally unified diagnostic and scoring criteria in the future.

The combination of laparoscopy and histological verification is considered as a standard diagnostic technique for endometriosis. Only three trials demonstrated that all of the patients were diagnosed with laparoscopy. In other trials, some included in this review used a clinical diagnostic standard and others employed laparoscopic diagnosis. However, we were unable to divide laparoscopic- and clinicaldiagnosed cases into two subgroups for sensitivity analysis because counting all of the cases of laparoscopic diagnosis in one study is impossible. 


\section{Conclusion}

Our study provided evidence that BSHXP is effective and safe for endometriosis, but this evidence is inconclusive because of the low methodological quality of the included RCTs. Our findings suggest that BSHXP is an alternative drug for endometriosis, but it should be further examined in future clinical research.

\section{Conflicts of Interest}

The authors declare that there are no conflicts of interest regarding the publication of this paper.

\section{Authors' Contributions}

Jing Shan, Wen Cheng, Dong-xia Zhai, Dan-ying Zhang, Rui-pin Yao, Ling-ling Bai, Zai-long Cai, Yu-huan Liu, and Chao-qin Yu had full access to all study data and take responsibility for its integrity and the accuracy of the analysis. Jing Shan, Zai-long Cai, and Chao-qin Yu were responsible for the study concept and design. Jing Shan and Wen Cheng were responsible for data acquisition. Dong-xia Zhai and Dan-ying Zhang were responsible for data extraction. The assessment of bias risk was performed by Jing Shan and Ruipin Yao; data analysis and interpretation were performed by Jing Shan, Ling-ling Bai, and Chao-qin Yu. Jing Shan drafted the paper, which was revised by Zai-long Cai and Chaoqin Yu. Statistical analyses were performed by Jing Shan and Wen Cheng. Zai-long Cai, Yu-huan Liu, and Chao-qin Yu supervised the study.

\section{Acknowledgments}

This study was supported by a grant from the National Science Foundation (NSFC) of China (no. 81573755 to Chaoqin Yu, 81503604 to Wen Cheng).

\section{References}

[1] M. Hickey, K. Ballard, and C. Farquhar, "Endometriosis," British Medical Journal, vol. 348, Article ID g1752, 2014.

[2] M. A. Wilbur, I.-M. Shih, J. H. Segars, and A. N. Fader, "Cancer Implications for Patients with Endometriosis," Seminars in Reproductive Medicine, vol. 35, no. 1, pp. 110-116, 2017.

[3] H. S. Kim, T. H. Kim, H. H. Chung, and Y. S. Song, "Risk and prognosis of ovarian cancer in women with endometriosis: a meta-analysis," British Journal of Cancer, vol. 110, no. 7, pp. 18781890, 2014.

[4] R. Z. Spaczynski and A. J. Duleba, "Diagnosis of endometriosis," Seminars in Reproductive Medicine, vol. 21, no. 2, pp. 193-208, 2003.

[5] K. E. Nnoaham, L. Hummelshoj, P. Webster et al., "Impact of endometriosis on quality of life and work productivity: a multicenter study across ten countries," Fertility and Sterility, vol. 96, no. 2, pp. 366.e8-373.e8, 2011.

[6] L. C. Giudice and L. C. Kao, "Endometriosis," The Lancet, vol. 364, no. 9447, pp. 1789-1799, 2004.

[7] S. H. Kim, H. D. Chae, C. H. Kim, and B. M. Kang, "Update on the treatment of endometriosis," Clinical and Experimental Reproductive Medicine, vol. 40, pp. 55-59, 2013.
[8] L. Goenka, M. George, and M. Sen, "A peek into the drug development scenario of endometriosis - A systematic review," Biomedicine \& Pharmacotherapy, vol. 90, pp. 575-585, 2017.

[9] A. D. Greene, S. A. Lang, J. A. Kendziorski, J. M. Sroga-Rios, T. J. Herzog, and K. A. Burns, "Endometriosis: Where are we and where are we going?" Reproduction, vol. 152, no. 3, pp. R63-R78, 2016.

[10] G. A. J. Dunselman, N. Vermeulen, C. Becker et al., "ESHRE guideline: management of women with endometriosis," Human Reproduction, vol. 29, no. 3, pp. 400-412, 2014.

[11] N. Berlanda, E. Somigliana, P. Viganò, and P. Vercellini, "Safety of medical treatments for endometriosis," Expert Opinion on Drug Safety, vol. 15, no. 1, pp. 21-30, 2016.

[12] S. Y. Park, S. H. Kim, H. D. Chae, C.-H. Kim, and B. M. Kang, "Efficacy and safety of dienogest in patients with endometriosis: A single-center observational study over 12 months," Clinical and Experimental Reproductive Medicine, vol. 43, no. 4, pp. 215220, 2016.

[13] Practice Committee of the American Society for Reproductive Medicine, "Treatment of pelvic pain associated with endometriosis," Fertility and Sterility, vol. 90, no. 5, pp. S260S269, 2008.

[14] R.-C. Fang, Y.-T. Tsai, J.-N. Lai, C.-H. Yeh, and C.-T. Wu, "The traditional chinese medicine prescription pattern of endometriosis patients in Taiwan: A population-based study," Evidence-Based Complementary and Alternative Medicine, vol. 2012, Article ID 591391, pp. 1-9, 2012.

[15] N. L. Zhang, C. Fu, T. F. Liu et al., "A data-driven method for syndrome type identification and classification in traditional Chinese medicine," Journal of Integrative Medicine, vol. 15, no. 2, pp. 110-123, 2017.

[16] J. Han, Q. Zhou, D. Zhang et al., "Adhesive and Invasive Effects and Mechanism of Neiyi Recipe On the Endometriosis," Chinese Journal of Integrated Traditional and Western Medicine, vol. 27, no. 1, pp. 87-88, 2007.

[17] H. Zhou, Q. Cong, Q. Wu et al., "Effect of Reinforcing Kidney and Activating Blood Therapy on Apoptosis Relevant Gene Bcl2/Bax Expression of Rat Models with Endometriosis," Journal of Zhejiang university of Traditional Chinese Medicine, vol. 34 , no. 6, pp. 830-835, 2010.

[18] D. Moher, A. Liberati, and J. Tetzlaff, "Preferred reporting items for systematic reviews and meta-analyses: the PRISMA statement," Journal of Clinical Epidemiology, vol. 62, no. 10, pp. 1006-1012, 2009.

[19] J. P. T. Higgins and S. Green, "Cochrane handbook for systematic reviews of interventions, version 5.1.0," in The Cochrane Collaboration, 2011, http://www.cochrane-handbook.org.

[20] Z. Ding and F. Lian, "Traditional Chinese medical herbs staged therapy in infertile women with endometriosis: A clinical study," International Journal of Clinical and Experimental Medicine, vol. 8, no. 8, pp. 14085-14089, 2015.

[21] H. Zhou and Q. Cong, "The immediate effect on blood pressure of acupuncture at Taichong (LR 3) in 65 cases of hypertension patient with hyperactivity of liver-yang," Journal of Traditional Chinese Medicine, vol. 49, no. 7, pp. 618-621, 2008.

[22] C.-L. Wei, "Effect of Bushen Huoxue Huayu Decoction on Clinical Efficacy and Tumor Necrosis Factor in Patients with Endometriosis," Guangming Journal of Chinese Medicine, vol. 26, no. 2, pp. 261-262, 2011.

[23] Y.-J. Shi, X.-T. Zou, and Y.-J. Ma, "Clinical observation on treatment of endometriosis with kidney deficiency and blood stasis 
type by traditional chinese medicine bushen quyu decoction," Journal of Frontiers of Medicine, vol. 5, no. 18, pp. 298-299, 2015.

[24] L. Mei, "Clinical observation of 30 cases of Ovarian Endometriosis Treated with Bushen Huoxue Decoction," Jiangsu Journal of Traditional Chinese Medicine, vol. 48, no. 1, pp. 4044, 2016.

[25] X.-W. Xu and T.-O. Liu, "Treatment of 52 Cases of Endometriosis with Bushen Huoxue Decoction," Shanxi Journal of Traditional Chinese Medicine, vol. 29, no. 3, pp. 266-288, 2008.

[26] W. Xia and L. Qin, “Treatment of 30 Cases of Endometriosis with Bushen Huoxue Decoction," TCM Research, vol. 21, no. 10, 2008.

[27] X.-L. Li, "Clinical Study on the effect of Kang Zheng Xiao Yi Granule In Treating Endometriosis," Gansu Journal of Traditional Chinese Medicine, vol. 18, no. 6, pp. 28-29, 2005.

[28] G.-M. Zhang and W.-Q. Zhang, "Effect of Kidney Warming, Quyu and Elmi inating Mass Method on the Cyclic Nucleotides and Immune Function of Endometriosis Patients," Chinese Archives of Traditional Chinese Medicine, vol. 28, no. 6, pp. 11441146, 2010.

[29] X.-T. Shen, Efficacy of Wenshen Xiaozheng Decoction on the kidney deficiency and blood stasis type of ovarian endometriomas and preliminary exploration of the possible mechanism [MSc. thesis], Nanjing University of Traditional Chinese Medicine, Nanjing, China, 2015.

[30] X.-L. Zhao, Clinical Study on the Effect of Yi Shen Xiao Zheng Decoction In Treating Endometriosis [MSc. thesis], Shandong University of Traditional Chinese Medicine, Jinan, China, 2008.

[31] L. Jian, X. Li, and X. Hu, "Clinical Observations on Treatment of Endometriosis by Tonifying Kidney and Removing Blood Stasis," Chinese Journal of Integrative Traditional and Western Medicine, vol. 18, no. 3, pp. 145-147, 1998.

[32] A.-P. Li and Y. Yu, "Treatment of 62 Cases of Endometriosis with Bushen Warming by Tonifying Kidney," Zhejiang Journal of Traditional Chinese Medicine, vol. 42, no. 7, p. 44, 2007.

[33] M. A. Bedaiwy, C. Allaire, P. Yong, and S. Alfaraj, "Medical Management of Endometriosis in Patients with Chronic Pelvic Pain," Seminars in Reproductive Medicine, vol. 35, no. 1, Article ID 001044, pp. 038-053, 2017.

[34] J. Donnez, R. N. Taylor, and H. S. Taylor, "Partial suppression of estradiol: a new strategy in endometriosis management?" Fertility and Sterility, vol. 107, no. 3, pp. 568-570, 2017.

[35] X. Gao, J. Outley, M. Botteman, J. Spalding, J. A. Simon, and C. L. Pashos, "Economic burden of endometriosis," Fertility and Sterility, vol. 86, no. 6, pp. 1561-1572, 2006.

[36] S. Simoens, L. Hummelshoj, and T. D’Hooghe, "Endometriosis: cost estimates and methodological perspective," Human Reproduction Update, vol. 13, no. 4, pp. 395-404, 2007.

[37] L. Fedele and N. Berlanda, "Emerging drugs for endometriosis," Expert Opinion on Emerging Drugs, vol. 9, no. 1, pp. 167-177, 2004.

[38] R. F. Casper, "Introduction: A focus on the medical management of endometriosis," Fertility and Sterility, vol. 107, no. 3, pp. 521-522, 2017.

[39] T. M. D’Hooghe, “Immunomodulators and aromatase inhibitors: are they the next generation of treatment for endometriosis?" Currebt Opinion in Obstetrics Gynecology, vol. 15, pp. 243-249, 2003.

[40] J.-T. Zhang, S.-Y. Du, and N. Wang, "Effect of Bushen Tiaojing Decoction on nephrasthenia anovulatory infertility," Chinese of Journal Family Planning, vol. 22, no. 7, pp. 460-464, 2014.
[41] F. Lian, H.-C. Wu, Z.-G. Sun, Y. Guo, L. Shi, and M.-Y. Xue, "Effects of Liuwei Dihuang Granule on the outcomes of in vitro fertilization pre-embryo transfer in infertility women with Kidney-yin deficiency syndrome and the proteome expressions in the follicular fluid," Chinese Journal of Integrative Medicine, vol. 20, no. 7, pp. 503-509, 2014.

[42] L. Fang, H.-P. Liu, Y.-X. Wang et al., "Study on Mechanism of Quyuj iedu Method in Treating Endometriosis of Stasis-toxic Syndrome Type," Chinese Journal of Integrative Medicine, vol. 26, no. 2, pp. 110-113, 2006.

[43] Q. Zhang, X. Liu, and S.-W. Guo, "Progressive development of endometriosis and its hindrance by anti-platelet treatment in mice with induced endometriosis," Reproductive BioMedicine Online, vol. 34, no. 2, pp. 124-136, 2017.

[44] L. Xu, Q.-L. Zhou, J. Han et al., "Effects of Chinese herbal medicine Neiyi Recipe-medicated serum on angiopoiesis of endometriosis in the chick chorioal lantoic membrane model," Journal of Chinese Integrative Medicine, vol. 10, no. 7, pp. 800806, 2012. 


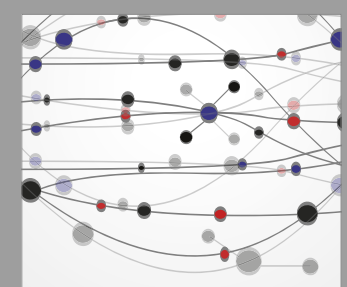

The Scientific World Journal
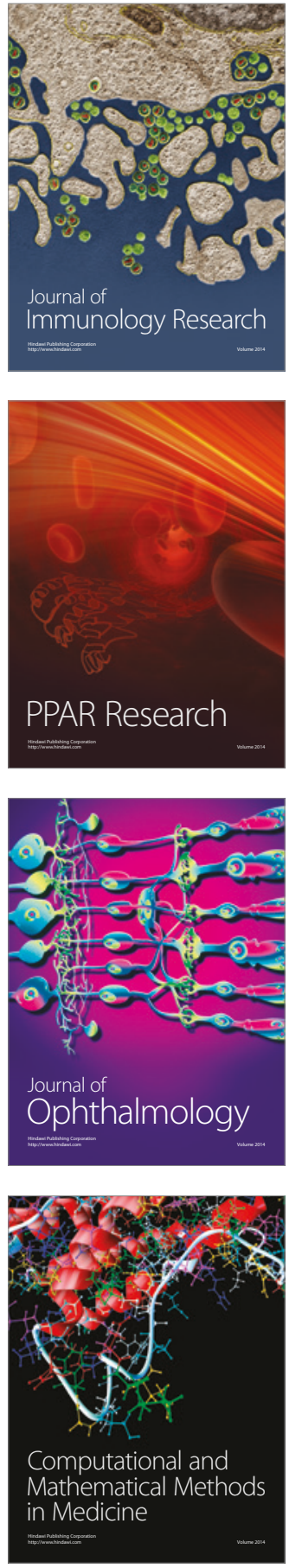

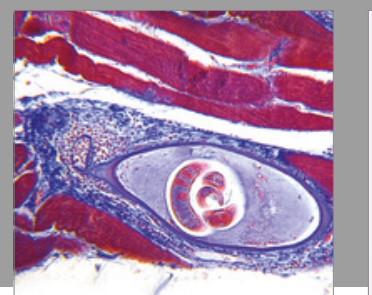

Gastroenterology Research and Practice
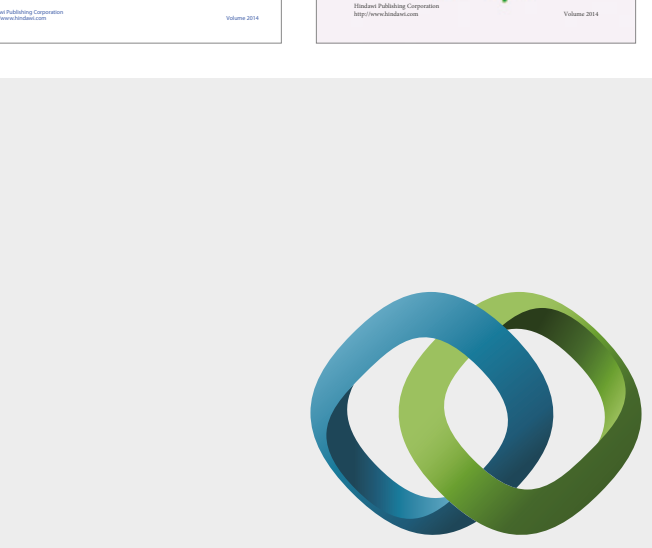

\section{Hindawi}

Submit your manuscripts at

https://www.hindawi.com
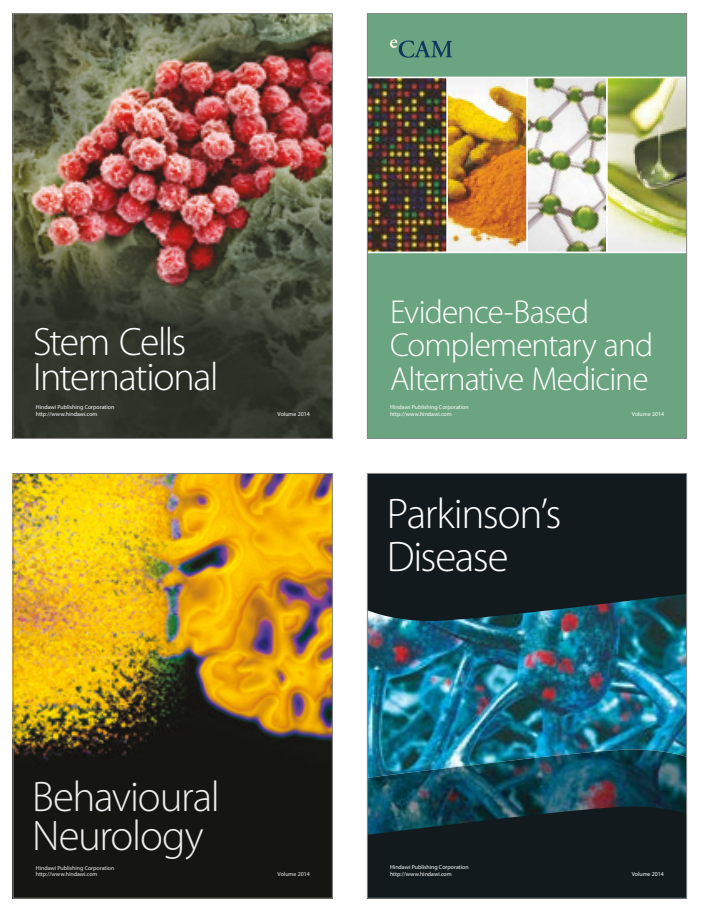
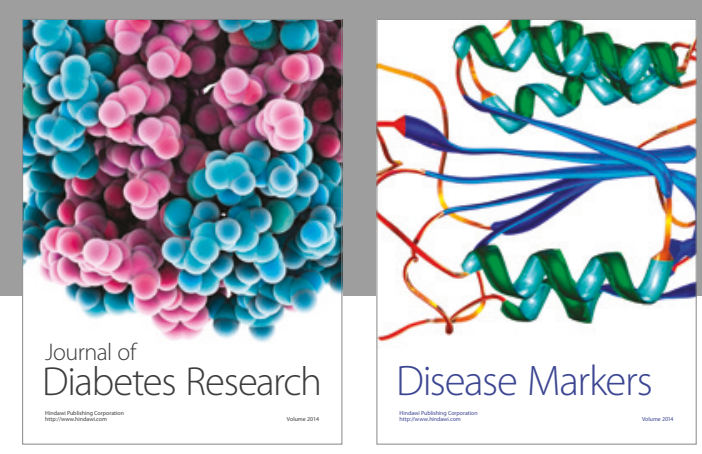

Disease Markers
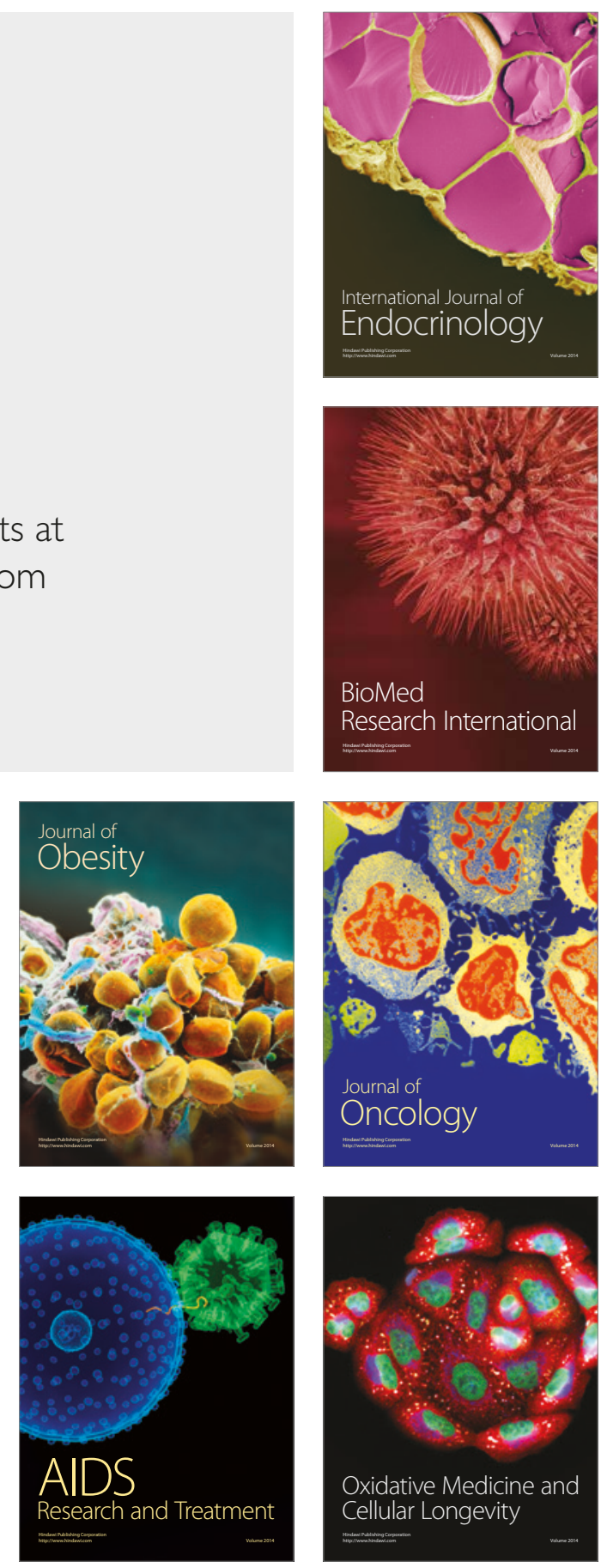